\title{
Improved gastric emptying in diabetic rats by irbesartan via decreased serum leptin and ameliorated gastric microcirculation
}

\author{
L. He, Y. Sun, Y. Zhu, R. Ren, Y. Zhang and F. Wang \\ Department of Digestive Diseases, \\ The Second Hospital of Hebei Medical University, \\ Shijiazhuang, China \\ Corresponding author: Y.F. Sun \\ E-mail: yufengsun@yeah.net
}

Genet. Mol. Res. 13 (3): 7163-7172 (2014)

Received May 23, 2013

Accepted January 23, 2014

Published September 5, 2014

DOI http://dx.doi.org/10.4238/2014.September.5.2

\begin{abstract}
Diabetic gastroparesis (DG) is a common clinical complication of diabetes mellitus. Leptin may cause delayed gastric emptying in the central and peripheral pathways. Microcirculatory disturbances in the stomach make gastric smooth muscles and nerves hypoxic-ischemic, thereby impairing gastric motility. Irbesartan is an angiotensin II (ATII) receptor blocker that indirectly decreases serum leptin levels and improves blood vessel endothelia. This study examined the effect of irbesartan on DG and its relationship with serum leptin levels and microcirculatory disturbances of the stomach. SpragueDawley rats were injected with streptozotocin to induce diabetes and were then treated with or without $0.012 \mathrm{~g} \cdot \mathrm{kg}^{-1} \cdot \mathrm{d}^{-1}$ irbesartan by gavage. After six weeks of treatment, the gastric evacuation rate (GER) was measured using phenol red. Serum leptin levels were detected using enzyme-linked immunosorbent assays. Endothelin (ET) in the stomach tissue was examined using a radioimmunoassay, whereas chemical colorimetry was used to measure the nitric oxide synthase (NOS) activity of stomach tissues. The mRNA expression of the ATII
\end{abstract}


receptor (AT1R) was assessed using reverse transcription-polymerase chain reaction. Treatment with irbesartan significantly increased the GER of diabetic rats and reduced the serum leptin levels, as well as decreased the ET content and AT1R mRNA expression in the stomach $(\mathrm{P}<0.05)$. Changes in the cNOS activity after irbesartan intervention were not significant $(\mathrm{P}>0.05)$, whereas iNOS activity was significantly decreased $(\mathrm{P}<0.05)$. Irbesartan can alleviate hyperglycemia-induced delayed gastric emptying, which is associated with decreased serum leptin levels and improved microcirculation in the stomach.

Key words: Diabetes mellitus; Diabetic gastroparesis; Irbesartan; Gastric evacuation rate; Leptin; Endothelin

\section{INTRODUCTION}

Gastroparesis is characterized by a delay in the emptying of stomach contents in the absence of mechanical obstructions in the stomach. The gastrointestinal symptoms of delayed gastric emptying are nonspecific, including early satiety, nausea, vomiting, regurgitation, fullness, and bloating (Horowitz et al., 2002). The most common known underlying cause of gastroparesis is diabetes mellitus (Alam et al., 2010). Previous studies showed that approximately 30 to $50 \%$ of people with diabetes types 1 and 2 exhibit delayed gastric emptying (Patrick and Epstein, 2008; Samsom et al., 2009). Moreover, delayed gastric emptying occurs in up to $50 \%$ of patients with chronic diabetes, and is thereby associated with significantly impaired quality of life and diabetic control (Murray et al., 2005). To date, the pathogenesis of diabetic gastroparesis (DG) is considered to be closely related to diabetic autonomic neuropathy, gastrointestinal hormonal abnormalities, gastrointestinal ischemia, and hyperglycemia (Samsom et al., 2009). However, DG remains difficult to treat effectively. Gastroparesis may cause intractable vomiting and an inability to feed, which indicate a poor prognosis (Horowitz et al., 2001). Thus, the study of DG treatment has an important practical significance.

At present, management methods for DG involve the empirical use of prokinetic drugs such as domperidone, metoclopramide, cisapride, and erythromycin. These drugs have motilin analogue properties that are useful in a subset of patients. However, the effects of these drugs are unpredictable. Their short-term administration may accelerate gastric emptying but will not necessarily improve its symptoms. Furthermore, these short-term benefits are frequently lost with chronic administration (Murray et al., 2005). Irbesartan is a basic drug for the prophylaxis and treatment of diabetic nephropathy and hypertension. In this study, we investigated if irbesartan could be used to prevent and cure DG.

Patients with diabetes mellitus have an abnormally active renin-angiotensin system (RAS), with angiotensin II (ATII) being the most bioactive substance. The combined action of the gastrointestinal hormone leptin and its receptors may cause delayed gastric emptying through central and peripheral pathways (Waelput et al., 2002; Monteleone et al., 2004). ATII stimulates adipocytes to synthesize and secrete leptin. However, ATII may induce hypertrophy in glomerular cells, the proliferation of mesangial cells, and the increased levels of extracellular base proteins (Lacourcière et al., 2000). Consequently, a metabolic decrease of leptin occurs in the kidney.

ATII receptor blockers reduce or mitigate the synthesis and effect of ATII, which re- 
strains the synthesis and secretion of leptin (Cassis et al., 2004). Irbesartan is a non-peptide chronic ATII receptor antagonist that protects against diabetic nephropathy (DN) (Liu et al., 2003), aside from its above-mentioned effect. Therefore, irbesartan could indirectly reduce serum leptin levels. Irbesartan improves the blood vessel endothelia, which contributes toward reducing microvascular complications by decreasing the endothelin (ET) level and increasing the nitric oxide (NO) level (Candido et al., 2004).

Microangiopathy is a risk factor for diabetic complications and is visible in DN, retinopathy, and neuropathy, resulting in pathological changes in the alimentary tract. Microcirculatory disturbances in the stomach make gastric smooth muscles and nerves hypoxic-ischemic, which affects the functions of the diastole and the contraction of gastric smooth muscles, and eventually impairs gastric emptying. An impaired blood vessel endothelium is a major pathological change that causes microcirculation disturbances. ET and NO are important indices of the blood vessel endothelium status. These compounds have contrasting effects on blood vessels. The former causes vessels to contract, which may be harmful to health, whereas the latter causes vessels to relax, thereby providing a protective effect. Thus, a dynamic balance between ET and NO is important to maintain a normal blood vessel endothelium (Kawanabe and Nauli, 2011).

This study aimed to investigate the mechanism by which irbesartan improves the impaired gastric emptying of DG rats. Changes in the serum leptin levels as well as the expression levels of ET, NO synthase (NOS), and AT1R mRNA were observed. ATII has biological functions when it binds to AT1R. Thus, the expression of AT1R can be used to determine the biological activity of ATII.

\section{MATERIAL AND METHODS}

\section{Animal models for diabetes}

Thirty healthy five-week-old male Sprague-Dawley (SD) rats (SPF-grade) were used. Each rat weighed approximately $200 \mathrm{~g}$. The rats were provided by the Hebei Medical University Laboratory Animal Center (Animal Certificate No. 1112061).

During the experiment, food and water were supplied ad libitum. The rats were given regular feed, which was supplied by the Hebei Medical University Laboratory Animal Center. The animals were housed at 20 to $24^{\circ} \mathrm{C}$ in independently ventilated cages with a $12 \mathrm{~h}$ light: 12 $\mathrm{h}$ dark photoperiod.

The rats were allowed to adapt to their feed for four weeks before they were randomly divided into two groups. The normal control (NC) group was composed of 10 individuals. Based on our preliminary studies, the experimental group included 20 diabetic rats that were intraperitoneally injected with $50 \mathrm{mg} / \mathrm{kg}$ streptozotocin (STZ; Sigma Chemical; St. Louis, USA) in $0.1 \mathrm{M}$ citrate buffer, $\mathrm{pH} 4.4$, to induce diabetes. The control group was injected with citrate buffer alone. After $72 \mathrm{~h}$, we collected intravenous blood samples from all rats to measure their blood glucose levels. Diabetes was successfully modeled when the random blood glucose concentration exceeded $16.19 \mathrm{mM}$.

\section{Drug intervention and sampling}

After the diabetic model was successfully established, the experimental group was randomly divided into the diabetes mellitus control (DC) and the irbesartan-treated control 
(IC) groups, with 10 rats in each group. Irbesartan (Sanofi fort SAN Della Minsheng Pharmaceutical Co., Ltd.; Hangzhou, China) at a dose of $0.012 \mathrm{~g} / \mathrm{kg}$ body weight was intragastrically administered to the IC group, according to the surface area conversion. The normal and DC groups were given the same amount of saline by gavage daily at ten o'clock in the morning for six weeks. We collected blood samples at the end of drug intervention to measure the gastric emptying rate (GER). The stomachs of the rats were harvested and stored at $-80^{\circ} \mathrm{C}$ for measurement of the ET content, NOS activity, and AT1R mRNA expression.

\section{Observation of general indices}

All experimental rats were weighed at the end of drug intervention to compare the changes in their weight after the intervention. The blood glucose (BG), serum creatinine (Scr), serum total cholesterol (TC), and serum triglyceride (TG) levels were determined using an automatic analyzer (Beckman CX7; USA).

\section{GER measurement}

The protocol of GER measurement followed the methods described in Miyamoto et al. (2001). At the end of the sixth week of drug intervention, the rats were fasted for 16 to 24 $\mathrm{h}$ and were deprived of water for $2 \mathrm{~h}$. Subsequently, the rats were administered with $2 \mathrm{~mL} 500$ $\mathrm{mg} / \mathrm{L}$ phenol red solution by gavage. After $15 \mathrm{~min}$, the rats were intraperitoneally anesthetized with $80 \mathrm{~g} / \mathrm{L} 400 \mathrm{mg} / \mathrm{kg}$ chloral hydrate. To collect their stomachs, we performed a laparotomy on each rat, ligated the cardia and pylorus, removed the stomach, cut along the greater gastric curvature, and immediately rinsed the stomach with distilled water. The volume was fixed at $20 \mathrm{~mL}$. Subsequently, $20 \mathrm{~mL} 0.5 \mathrm{M}$ sodium hydroxide was added, and the suspension was mixed well. The mixture was allowed to stand for $1 \mathrm{~h}$ before $0.5 \mathrm{~mL} 20 \%$ dichloroisocyanuric acid was added to $5 \mathrm{~mL}$ supernatant to remove proteins. The mixture was centrifuged at 3500 rpm for $10 \mathrm{~min}$, and then the supernatant was collected. We measured the optical density of each sample using a spectrophotometer at a $560 \mathrm{~nm}$ wavelength (designated as the measured value). Alternatively, a $2 \mathrm{~mL}$ phenol red-methyl cellulose solution was mixed with $18 \mathrm{~mL}$ dis-

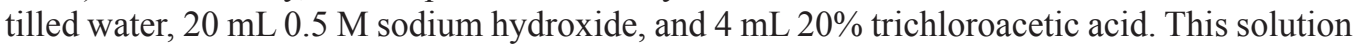
was mixed well and was used to measure optical density (designated as the standard phenol red optical density).

The rat GER was then computed using the following equation:

GER $=(1$ - measured optical density $) /$ standard phenol red optical density $) \times 100 \%$.

\section{Measurement of serum leptin levels and ET contents}

The blood serum leptin level and the ET content in the stomach tissue samples were determined using radioimmunoassay kits from the Huabulite Biological Technology Research Institute of Beijing, China.

First, we took $2 \mathrm{~mL}$ venous blood and injected each sample into a test tube containing $30 \mu \mathrm{L} \mathrm{7.5 \%} \mathrm{disodium} \mathrm{ethylenediaminetetraacetic} \mathrm{acid} \mathrm{(EDTA)} \mathrm{and} 40 \mu \mathrm{L}$ aprotinin. After the blood coagulated, we separated the serum by centrifugation at $3000 \mathrm{rpm}$ for $10 \mathrm{~min}$ at $4{ }^{\circ} \mathrm{C}$, and then the appropriate kit was used according to manufacturer instructions.

Second, the tissues maintained at $-80^{\circ} \mathrm{C}$ were used to measure the ET content. For 
each tissue sample, $1 \mathrm{~mL}$ saline was added before the tissues were homogenized. The homogenate was centrifuged at $3000 \mathrm{rpm}$ for $15 \mathrm{~min}$ at $4^{\circ} \mathrm{C}$ to obtain the supernatant. The appropriate detection kit was then used based on manufacturer instructions. Furthermore, the total protein in the stomach tissue was determined to calculate the ET content per mg protein. The Coomassie brilliant blue dye in the Bradford protein assay kit (Tiangen Biotech; Beijing) was used to measure the protein content in the stomach tissue.

\section{NOS activity}

NOS can catalyze the reaction of L-Arg and dioxygen to generate NO. When combined with nucleophilic substance, NO produces a colored compound that was measured using a spectrophotometer as the absorbance at a $560 \mathrm{~nm}$ wavelength. The protein content was divided by the volume $(\mathrm{mL})$ stomach supernatant. The specific steps were performed according to manufacturer instructions for the NOS detection kit (Jiancheng Institute of Biological Engineering; Nanjing, China).

\section{Semi-quantitative reverse transcription-polymerase chain reaction (RT-PCR)}

The total cellular RNA was prepared from the stomach tissue using Trizol according to manufacturer instructions (Invitrogen; Carlsbad, USA). Single-stranded cDNA was synthesized from individual $2 \mu \mathrm{g}$ samples total RNA using the TUREscript 1st Strand cDNA Synthesis Kit (Tiangen). After the addition of $0.4 \mu \mathrm{M}$ of each pair of primers and the template DNA to the master mix, RT-PCR was performed using the 2X Tap PCR MasterMix Kit (Tiangen). The following PCR protocol was used: initial denaturation at $94^{\circ} \mathrm{C}$ for $3 \mathrm{~min}$, followed by 35 cycles of denaturation at $94^{\circ} \mathrm{C}$ for $40 \mathrm{~s}$, annealing at $56^{\circ} \mathrm{C}$ for $50 \mathrm{~s}$, and extension at $72^{\circ} \mathrm{C}$ for $50 \mathrm{~s}$, with a final extension at $72^{\circ} \mathrm{C}$ for $10 \mathrm{~min}$. The PCR products were stored at $4^{\circ} \mathrm{C}$ until further use. The following oligonucleotide primer sequences were used: forward primer 5'-CCAGCGTCAGTTTCAATC-3' and reverse primer 5'-TAGGGCTTTCCAAATAAGAG TA-3' for AT1R; forward primer 5'-CCTTCATTGACCTCAACTAC-3' and reverse primer 5'-GGAAGGCCATGCCAGTGAGC-3' for $\beta$-actin. To visualize the level of gene expression, the individual PCR products were separated by electrophoresis on $1 \%$ agarose gel and stained with ethidium bromide. The signal intensity of the AT1R mRNA was normalized to that of the housekeeping $\beta$-actin gene. The results were expressed as the ratio of the intensity of AT1R expression to that of $\beta$-actin.

\section{Statistical analysis}

The data measured, which are presented as means and standard deviations, followed a normal distribution and homogeneity of variance. Differences between groups were compared using one-way analysis of variance (ANOVA). If the difference was statistically significant, we further compared the groups selected using the Student Newman-Keuls test. All of the abovementioned tests were two-tailed, and $\mathrm{P}<0.05$ was considered to be statistically significant. Nonparametric alternatives, namely the Wilcoxon signed rank test and the Kruskal-Wallis test with the Dunn's post-hoc test, were used when the data did not meet the criteria for parametric analyses. 


\section{RESULTS}

\section{Comparison of rat characteristics}

$\mathrm{BG}, \mathrm{TG}$, and TC were all higher in diabetic rats than in the non-diabetic controls $(\mathrm{P}<$ 0.05 ; Table 1). No significant differences were observed between the diabetes control group and the irbesartan-treated group for the above-mentioned parameters $(\mathrm{P}>0.05$; Table 1). The weight of all diabetic rats were reduced compared with normal controls $(\mathrm{P}<0.05$; Table 1$)$, whereas the irbesartan-treated rats weighed more than the diabetes controls $(\mathrm{P}<0.05$; Table 1$)$. Scr was significantly affected in diabetic rats after treatment with irbesartan. Compared with the diabetes control group, the Scr level of the irbesartan-treated group was reduced $(\mathrm{P}<0.05$; Table 1$)$.

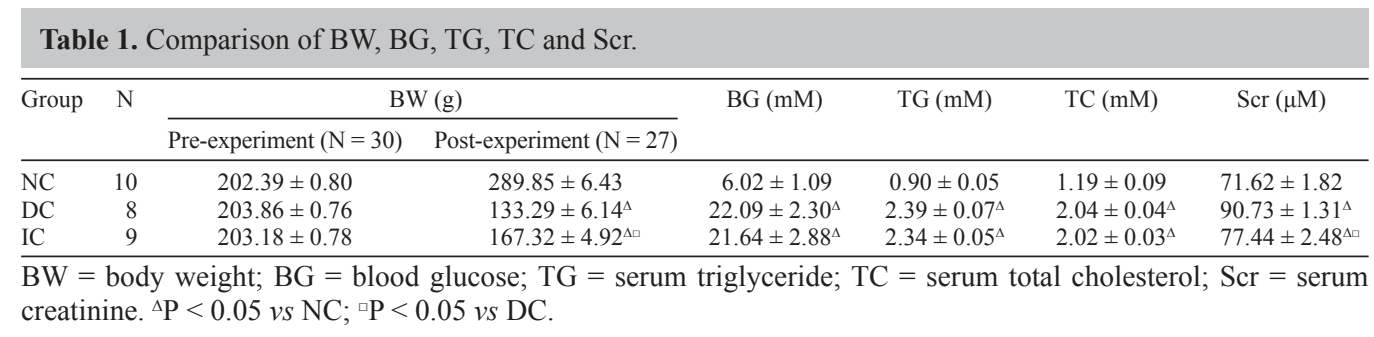

\section{Gastric emptying}

Six weeks after STZ treatment, gastric emptying was significantly reduced in the diabetes control group compared with the normal control group $(\mathrm{P}<0.05$; Table 2$)$. The GER was significantly accelerated in the irbesartan-treated group compared with that of the diabetes control group $(\mathrm{P}<0.05$; Table 2$)$, whereas their GERs were still lower than those of normal controls $(\mathrm{P}<0.05$; Table 2$)$.

\section{Table 2. Comparision of GER, ET, NOS, AT1R mRNA.}

\begin{tabular}{|c|c|c|c|c|c|c|}
\hline Group & $\mathrm{N}$ & GER (\%) & ET (pg/mg protein) & iNOS (pg/mg protein) & cNOS (pg/mg protein) & AT1R/ $\beta$-actin ratio \\
\hline $\mathrm{NC}$ & 10 & $62.64 \pm 4.51$ & $18.520 \pm 2.795$ & $0.209 \pm 0.015$ & $0.521 \pm 0.057$ & $0.207 \pm 0.004$ \\
\hline $\mathrm{DC}$ & 8 & $18.65 \pm 4.30^{\Delta}$ & $26.850 \pm 2.897^{\Delta}$ & $0.276 \pm 0.021^{\Delta}$ & $0.323 \pm 0.079^{\Delta}$ & $0.741 \pm 0.010^{\Delta}$ \\
\hline IC & 9 & $22.64 \pm 2.88^{\Delta \square}$ & $21.660 \pm 4.686^{\Delta \square}$ & $0.246 \pm 0.033^{\Delta \square}$ & $0.384 \pm 0.067^{\Delta}$ & $0.546 \pm 0.005^{\Delta \square}$ \\
\hline
\end{tabular}

\section{Serum leptin concentrations}

In all diabetic rats, the serum leptin level was higher than that in normal controls $(\mathrm{P}<$ 0.05; Table 2). However, the serum leptin levels of diabetic rats were markedly decreased after treatment with irbesartan $(\mathrm{P}<0.05$; Table 2).

\section{ET content and NOS activity}

A radioimmunoassay was used to determine the ET content. Results showed that the 
ET levels of the two diabetic groups were notably increased compared with that of the normal control group $(\mathrm{P}<0.05$; Table 2$)$. After irbesartan intervention, the ET level was significantly lower in the irbesartan-treated group than in the diabetes control group $(\mathrm{P}<0.05$; Table 2$)$.

Chemical colorimetry showed that in all diabetic rats, the iNOS activity significantly increased, whereas the cNOS activity was markedly decreased, compared with those of normal controls $(\mathrm{P}<0.05$; Table 2$)$. However, in the irbesartan-treated group, iNOS activity significantly declined $(\mathrm{P}<0.05$; Table 2$)$. There were no significant changes in the cNOS activity between the irbesartan-treated group and the diabetes control group $(\mathrm{P}>0.05$; Table 2$)$.

\section{AT1R mRNA expression}

The expression of AT1R mRNA in the stomachs of all diabetic rats was significantly upregulated compared to that of the normal control group $(\mathrm{P}<0.05$; Table 3, Figure 1$)$. When treatment with irbesartan was complete, the AT1R mRNA expression level was notably decreased $(\mathrm{P}<0.05$; Table 3, Figure 1).

Table 3. ET content, NOS activity AT1R mRNA expression in stomach tissue.

\begin{tabular}{lrcccc}
\hline Group & $\mathrm{N}$ & ET (pg/mg protein) & iNOS (pg/mg protein) & cNOS (pg/mg protein) & AT1R/ $\beta$-actin ratio \\
\hline Normal & 10 & $18.520 \pm 2.795$ & $0.209 \pm 0.015$ & $0.521 \pm 0.057$ & $0.207 \pm 0.004$ \\
Mellitus & 8 & $26.850 \pm 2.897^{\Delta}$ & $0.276 \pm 0.021^{\Delta}$ & $0.323 \pm 0.079^{\Delta}$ & $0.741 \pm 0.010^{\Delta}$ \\
Irbesartan & 9 & $21.660 \pm 4.686^{\Delta \square}$ & $0.246 \pm 0.033^{\Delta \square}$ & $0.384 \pm 0.067^{\Delta \Delta}$ & $0.546 \pm 0.005^{\Delta \square}$ \\
\hline
\end{tabular}

For abbreviations, see legend to Table $2 .{ }^{\Delta} \mathrm{P}<0.05$ vs normal; ${ }^{\circ} \mathrm{P}<0.05$ vs mellitus; ${ }^{\wedge} \mathrm{P}>0.05$ vs mellitus.
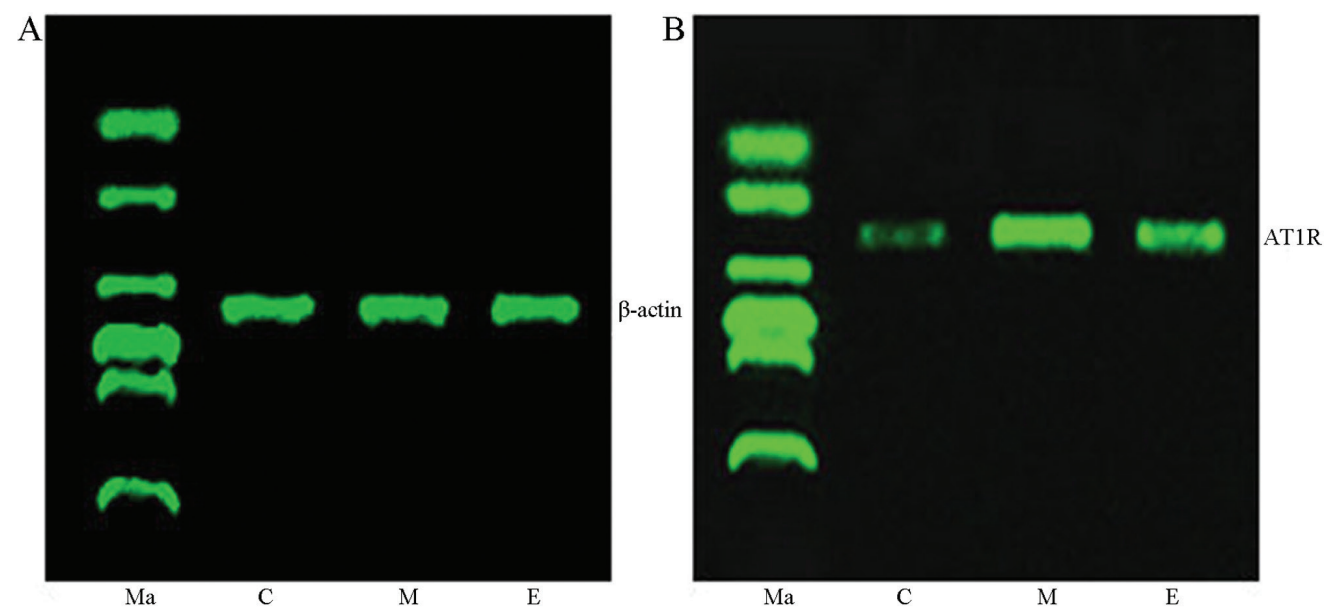

Figure 1. Expression of $\beta$-actin and AT1R mRNA examined by RT-PCR. A. $\beta$-actin; B. AT1R. Lane Ma = DNA marker, from top to bottom as follows: 100, 250, 500, 750, 1000, $2000 \mathrm{bp}$; lane $C=$ control group; lane $M=$ model group; lane $E$ = Irbesartan-treated group.

\section{DISCUSSION}

We proposed and demonstrated, for the first time, that irbesartan could improve gastric emptying of DG rats to some extent. Data in this experiment showed that after irbesartan 
intervention, the GER of all diabetic rats significantly decreased, although the GER of the irbesartan-treated group was still higher than that of the diabetes control group.

We focused on two aspects to evaluate the possible mechanism for the action of irbesartan in DG. First, the effect might be mediated by the gastrointestinal hormone leptin, which is encoded by the $O b$ gene. Leptin is a polypeptide hormone that is secreted by fat cells and gastric mucosa epithelial cells (Cammisotto et al., 2005), and is closely related to gastric motility. Upon binding to its receptors in the hypothalamus, leptin induces the hypothalamicneuropeptide path. The said path primarily restrains the arcuate nucleus in the hypothalamus from expressing and secreting neuropeptide $\mathrm{Y}$, which consequently inhibits appetites and could result in weight loss (Matsuoka et al., 1997). Martínez et al. (1999) injected 3 mg leptin into the ventricle of model animals, which caused the cumulative food intake for $5 \mathrm{~h}$ to decrease by $39 \%$ and stomach transport to decrease by $50 \%$. Their study suggested that leptin partially influenced gastric emptying. Another study (Cakir et al., 2007) showed that after the right ventricle and stomach of rats were injected with leptin, gastric emptying was delayed, thereby demonstrating that leptin functions in gastric motility through a synergistic effect with cholecystokinin (CCK). Leptin stimulates the secretion of CCK, which activates the vagus and delays the signal transfer to the brain. Diabetes mellitus increases ATII activity, which can promote fat cells to secrete leptin and damage the renal hemodynamics, thereby decreasing leptin metabolism. Two large prospective, randomized, double-blinded clinical trials demonstrated that irbesartan prevented the onset (Lewis et al., 2001) and progression (Parving et al., 2001) of chronic kidney disease, independent of its blood pressure lowering effect. In our experiment, irbesartan decreased serum leptin levels. We can infer that irbesartan reduced or interdicted the synthesis and effect of ATII, which consequently restrained the synthesis and secretion of leptin (Cassis et al., 2004). Moreover, irbesartan can decrease Scr levels to protect the kidney and to increase leptin metabolism.

The other mechanism we evaluated was related to the microangiopathy of the stomach before the appearance of clinical digestive symptoms (Grover et al., 2011). Endothelial dysfunction is caused by diabetes and accelerates the progression of the disease. Therefore, the vessel endothelium is an important target tissue in diabetic patients. Research has shown that endothelial dysfunction was significantly improved by short-term atorvastatin and/or irbesartan treatment in type 2 diabetic patients without affecting blood pressure (Ceriello et al., 2005). Impaired endothelial function is the main pathological feature of diabetic microangiopathy (Rizzoni and Rosei, 2009). ET and NO are known indexes for the condition of the blood vessel endothelium. In the present study, the ET content and cNOS activity decreased in all diabetic rats, thereby indicating that diabetes mellitus can cause pathological changes in the blood vessel endothelium. After treatment with irbesartan, the ET level and iNOS activity that generated detrimental NO were both decreased. NO induces sulfhydryl oxidation and lipid peroxidation to cause endothelial cell injury. However, there were no significant changes in cNOS activity, although the reasons for this phenomenon are yet unknown. NO from cNOS maintains the vascular smooth muscles in a relaxed state, which maintains the stomach blood flow at a certain level to protect the mucous membrane (Kwiecien et al., 2008). Moreover, NO may limit the proliferation of vascular smooth muscles (Umar et al., 2010).

The physiological effects of ATII include vasoconstriction and triggering of the growth, migration, and apoptosis of cells (Touyz, 2005). ATII exerts physiological effects by binding to its receptors (mainly AT1R), which are found on vascular smooth muscle cells and endothelial 
cells (de Gasparo et al., 2000). We propose that irbesartan may improve the condition of the blood vessel endothelium by decreasing the expression of AT1R mRNA in the stomach tissue (as shown in our experiments). In addition, irbesartan had no effect on the BG, TG, and TC in this experiment, which contrasts with results of previous reports (Janiak et al., 2006; Shimamura et al., 2011). This difference was most likely observed because the dose of irbesartan in the present study was lower $(50 \mathrm{mg} / \mathrm{kg})$, and because the treatment period was shorter than those used in previous reports. However, the weight of the diabetic rats was increased, which was probably related to the improvement of gastric emptying to increase appetite.

In summary, we conducted preliminary studies on the effect of irbesartan on DG; however, whether irbesartan can be applied for the treatment of DG in clinical settings requires repeated animal experiments. Only a small dose of irbesartan was used in our short-term trials. If the dosage of irbesartan was increased and the test period was prolonged, the results may differ. Results from similar future studies will provide a solid theoretical foundation for treating diabetes mellitus with irbesartan.

\section{ACKNOWLEDGMENTS}

Research supported by the scientific research fund project of the Hebei Provincial Health Bureau (\#20110074).

\section{REFERENCES}

Alam U, Asghar O and Malik RA (2010). Diabetic gastroparesis: Therapeutic options. Diabetes Ther. 1: 32-43.

Cakir B, Kasimay O, Devseren E and Yegen BC (2007). Leptin inhibits gastric emptying in rats: role of CCK receptors and vagal afferent fibers. Physiol. Res. 56: 315-322.

Cammisotto PG, Renaud C, Gingras D, Delvin E, et al. (2005). Endocrine and exocrine secretion of leptin by the gastric mucosa. J. Histochem. Cytochem. 53: 851-860.

Candido R, Allen TJ, Lassila M, Cao Z, et al. (2004). Irbesartan but not amlodipine suppresses diabetes-associated atherosclerosis. Circulation 109: 1536-1542.

Cassis LA, English VL, Bharadwaj K and Boustany CM (2004). Differential effects of local versus systemic angiotensin II in the regulation of leptin release from adipocytes. Endocrinology 145: 169-174.

Ceriello A, Assaloni R, Da Ros R, Maier A, et al. (2005). Effect of atorvastatin and irbesartan, alone and in combination, on postprandial endothelial dysfunction, oxidative stress, and inflammation in type 2 diabetic patients. Circulation 111: 2518-2524.

de Gasparo M, Catt KJ, Inagami T, Wright JW, et al. (2000). International union of pharmacology. XXIII. The angiotensin II receptors. Pharmacol. Rev. 52: 415-472.

Grover M, Farrugia G, Lurken MS, Bernard CE, et al. (2011). Cellular changes in diabetic and idiopathic gastroparesis. Gastroenterology 140: 1575-1585.

Horowitz M, Su YC, Rayner CK and Jones KL (2001). Gastroparesis: prevalence, clinical significance and treatment. Can. J. Gastroenterol. 15: 805-813.

Horowitz M, O’Donovan D, Jones KL, Feinle C, et al. (2002). Gastric emptying in diabetes: clinical significance and treatment. Diabet. Med. 19: 177-194.

Janiak P, Bidouard JP, Cadrouvele C, Poirier B, et al. (2006). Long-term blockade of angiotensin AT1 receptors increases survival of obese Zucker rats. Eur. J. Pharmacol. 534: 271-279.

Kawanabe Y and Nauli SM (2011). Endothelin. Cell Mol. Life Sci. 68: 195-203.

Kwiecien S, Pawlik MW, Brzozowski T, Konturek PC, et al. (2008). Nitric oxide (NO)-releasing aspirin and (NO) donors in protection of gastric mucosa against stress. J. Physiol. Pharmacol. 59 (Suppl 2): 103-115.

Lacourcière Y, Bélanger A, Godin C, Hallé JP, et al. (2000). Long-term comparison of losartan and enalapril on kidney function in hypertensive type 2 diabetics with early nephropathy. Kidney Int. 58: 762-769.

Lewis EJ, Hunsicker LG, Clarke WR, Berl T, et al. (2001). Renoprotective effect of the angiotensin-receptor antagonist irbesartan in patients with nephropathy due to type 2 diabetes. N. Engl. J. Med. 345: 851-860. 
Liu BC, Chen Q, Luo DD, Sun J, et al. (2003). Mechanisms of irbesartan in prevention of renal lesion in streptozotocininduced diabetic rats. Acta Pharmacol. Sin. 24: 67-73.

Martínez V, Barrachina MD, Wang L and Tache Y (1999). Intracerebroventricular leptin inhibits gastric emptying of a solid nutrient meal in rats. Neuroreport 10: 3217-3221.

Matsuoka N, Ogawa Y, Hosoda K, Matsuda J, et al. (1997). Human leptin receptor gene in obese Japanese subjects: evidence against either obesity-causing mutations or association of sequence variants with obesity. Diabetologia 40: 1204-1210.

Miyamoto Y, Yoneda M, Morikawa A, Itoh H, et al. (2001). Gastric neuropeptides and gastric motor abnormality in streptozotocin-induced diabetic rats: observation for four weeks after streptozotocin. Dig. Dis. Sci. 46: 1596-1603.

Monteleone P, DiLieto A, Castaldo E and Maj M (2004). Leptin functioning in eating disorders. CNS Spectr. 9: 523-529.

Murray CD, Martin NM, Patterson M, Taylor SA, et al. (2005). Ghrelin enhances gastric emptying in diabetic gastroparesis: a double blind, placebo controlled, crossover study. Gut 54: 1693-1698.

Parving HH, Lehnert H, Brochner-Mortensen J, Gomis R, et al. (2001). The effect of irbesartan on the development of diabetic nephropathy in patients with type 2 diabetes. N. Engl. J. Med. 345: 870-878.

Patrick A and Epstein O (2008). Review article: gastroparesis. Aliment. Pharmacol. Ther. 27: 724-740.

Rizzoni D and Rosei EA (2009). Small artery remodeling in diabetes mellitus. Nutr. Metab. Cardiovasc. Dis. 19: 587-592.

Samsom M, Bharucha A, Gerich JE, Herrmann K, et al. (2009). Diabetes mellitus and gastric emptying: questions and issues in clinical practice. Diabetes Metab. Res. Rev. 25: 502-514.

Shimamura M, Nakagami H, Shimosato T, Moritani T, et al. (2011). Irbesartan improves endothelial dysfunction, abnormal lipid profile, proteinuria and liver dysfunction in Zucker diabetic fatty rats independent of glucose and insulin levels. Exp. Ther. Med. 2: 957-961.

Touyz RM (2005). Reactive oxygen species as mediators of calcium signaling by angiotensin II: implications in vascular physiology and pathophysiology. Antioxid. Redox Signal. 7: 1302-1314.

Umar S and van der Laarse A (2010). Nitric oxide and nitric oxide synthase isoforms in the normal, hypertrophic, and failing heart. Mol. Cell Biochem. 333: 191-201.

Waelput W, Brouckaert P, Broekaert D and Tavernier J (2002). A role for leptin in the systemic inflammatory response syndrome (SIRS) and in immune response. Curr. Drug Targets. Inflamm. Allergy 1: 277-289. 